\title{
DENUTRITION ET CANCERS DES VOIES AERO-DIGESTIVES SUPERIEURES
}

\author{
B. HAMMAMI, E. FOURATI, N. AYEDI, A. CHAKROUN, I. CHARFEDDINE, A. GHORBEL \\ SERVICE ORL ET CHIRURGIE CERVICO-FACIALE \\ CHU HABIB BOURGUIBA SFAX-TUNISIE
}

\begin{abstract}
RESUME
Les cancers des VADS ainsi que leur traitement sont responsables dans plus que $30 \%$ des cas de malnutrition pouvant être sévère et entraver la prise en charge de ces tumeurs. Cette mise au point a pour objectif de fournir un protocole de prise en charge nutritionnelle pratique des cancers des VADS.

Le diagnostic de malnutrition passe par un interrogatoire qui doit préciser essentiellement une perte de poids récente et essayer de la chiffrer. L'examen clinique est un temps capital dans le diagnostic et le classement de la sévérité de la dénutrition. La biologie permet également un diagnostic de sévérité mais aussi un moyen de surveillance fiable.

Dans la prise en charge de la dénutrition dans les cancers des VADS, il faut commencer très précocement avant même le diagnostic du cancer et le début du traitement. La voie orale doit toujours être privilégiée suivie de la voie entérale par sonde nasogastrique sinon à travers une gastrostomie ou jéjunostomie. L'alimentation parentérale garde une place limitée et de durée réduite.
\end{abstract}

\begin{abstract}
Head and neck cancer and its treatment are responsible in more than $30 \%$ of cases of malnutrition witch can be severe and interfere with the management of these tumors. The aim of this paper is to provide a protocol for nutritional management practices of these cancers.

The malnutrition diagnosis requires an examination that must define essentially a recent weight loss and try to quantify it. Clinical examination is a crucial time in the diagnosis and classification of malnutrition severity. Biology also provides a severity evaluation but also to follow up.

The management of malnutrition in head and neck cancer must be early before the cancer treatment. The oral nutrition support should always be preferred. Enteral nutritional support by nasogastric, gastrostomy or jejunostomy must be used if the oral support has failed. Parenteral nutrition retains a limited and short duration.
\end{abstract}

\section{INTRODUCTION}

Les cancers des voies aéro-digestives supérieures (VADS) et leurs traitements peuvent avoir des effets dévastateurs sur le statut nutritionnel du malade. Le traitement de ces cancers (chirurgie, chimiothérapie et radiothérapie) peut entraver de façon significative l'alimentation. Les séquelles thérapeutiques altèrent significativement la qualité de vie ce qui a un impact non négligeable sur le choix des nutriments. De ce fait, la dénutrition est un risque permanent et communément admis au cours des cancers des VADS, et représente même parfois le premier signe d'appel (1). La majorité des études rapportent un taux de 35 (2) à $50 \%$ (3) de dénutrition chez les malades porteurs d'un cancer des VADS. Elle nécessite donc d'être reconnue, prévenue si possible, et en tout cas prise en charge activement. Quel est l'état du sujet actuellement ? Et quelles sont les perspectives d'avenir ? Répondre à ces questions doit passer par la compréhension des mécanismes intimes de la dénutrition causée par le cancer des VADS ainsi que ses thérapies, la recherche des protocoles nutritionnels sans oublier la possibilité de thérapeutiques nutritionnelles spécifiques du cancer.
Cette mise au point a pour objectif de fournir un protocole de prise en charge nutritionnelle pratique des cancers des VADS.

\section{PHYSIOPATHOLOGIE}

$\Rightarrow$ Effet de la tumeur sur le statut nutritionnel

La perte pondérale est souvent le premier signe de la dénutrition et parfois même de la tumeur. Selon le type et le stade de la tumeur, la perte de poids a été rapportée dans 30 à $80 \%$ des cas et elle était sévère (perte > $10 \%$ du poids habituel) dans $15 \%$ des cas (4). Le statut nutritionnel peut être directement altéré par les troubles métaboliques induits par la tumeur. Le stade ultime de cette dénutrition est la cachexie qui est un état avancé de la malnutrition protido-calorique caractérisé par une perte de poids, fonte musculaire et dégradation de la qualité de vie. L'anorexie a été évoquée comme étant le facteur princeps à cette cachexie mais à elle seule elle ne peut pas induire une dégradation du statut nutritionnel. En pratique, il existe toujours un déséquilibre entre une diminution des apports et/ou des troubles de l'absorption des nutriments d'une part, des anomalies de la dépense éner- 
gétique d'origine métabolique, infectieuse, ou tumorale d'autre part (5).

En fait, la cellule tumorale produit plusieurs facteurs et stimule la synthèse de médiateurs ayant des effets sur les différents métabolismes du corps de l'hôte : médiateurs incluant les cytokines, neuropeptides et neurotransmetteurs (figure.1) $(5,7,8)$.

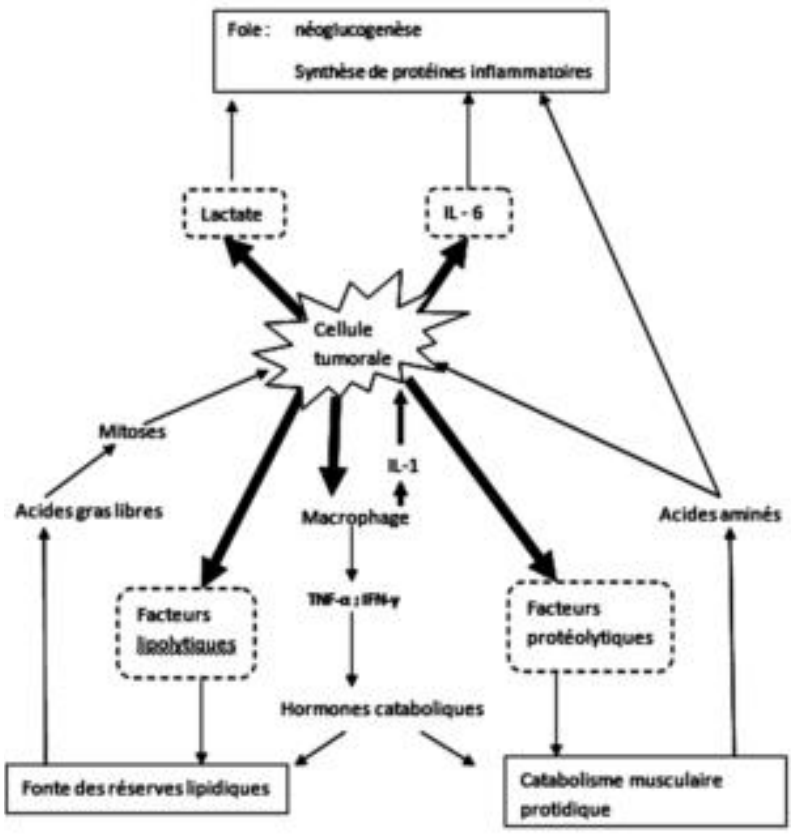

Figure 1 : Dysrégulation métabolique liée au cancer

L'altération du métabolisme de la graisse, des protéines et des carbohydrates est évidente dans les cancers avec cachexie (tableau I). La tumeur peut induire une déperdition de glucose et déclenche une oxydation de celui-ci et la conséquence est une augmentation de la glycolyse (8). Ainsi la perte de poids peut être due à la diminution d'apport énergétique, une augmentation de la consommation d'énergie ou la combinaison des deux.

\begin{tabular}{|c|c|c|}
\hline Métabolisme & Paramètre & Effets \\
\hline Protéique & $\begin{array}{c}\text { Flux corporel } \\
\text { Synthèse corporelle } \\
\text { Catabolisme corporel } \\
\text { Anabolisme musculaire } \\
\text { Catabolisme musculaire } \\
\text { Concentration en AA } \\
\end{array}$ & $\begin{array}{l} \pm \\
\pm \\
\lambda \\
y \\
\lambda \\
\pm \\
\end{array}$ \\
\hline Glucidique & $\begin{array}{c}\text { Néoglucogénèse } \\
\text { Flux } \\
\text { Recyclage } \\
\text { Sensibilité à l'insuline }\end{array}$ & $\begin{array}{l}\lambda \\
\lambda \\
\lambda \\
y\end{array}$ \\
\hline Lipidique & $\begin{array}{l}\text { Lipolyse } \\
\text { Lipogenèse }\end{array}$ & $\begin{array}{l}\lambda \\
?\end{array}$ \\
\hline Dépense énergétique & Dépense de fond & \pm \\
\hline
\end{tabular}

Tableau I: Effets métaboliques du cancer
Indépendamment des perturbations induites sur le métabolisme des tissus normaux, le tissu tumoral échappe à tous les mécanismes habituels de contrôle métabolique. D’une manière générale, la tumeur se développe quelque soit le niveau de dénutrition et d'anorexie et maintient un haut niveau d'activité métabolique aux dépens des tissus normaux (7).

\section{$\Rightarrow$ Implications nutritionnelles des traitements du cancer}

Le statut nutritionnel et le métabolisme de base au moment du diagnostic d'un cancer sont retenus par la majorité des auteurs comme étant des facteurs pronostiques. Ces facteurs peuvent également être discriminants dans l'indication de certains traitements. La chirurgie, la chimio et la radiothérapie peuvent avoir un effet direct (mécanique) et/ou indirect (métabolique) négatif sur le statut nutritionnel. Ces effets sont plus nocifs que la tumeur elle-même sur le statut nutritionnel (9). La réussite du traitement dépend de la tolérance du malade à terminer son traitement et ainsi il se trouve dans un cercle vicieux qui doit être cassé par une renutrition avant et pendant le traitement.

\section{- La chirurgie}

La chirurgie est souvent la première modalité thérapeutique des cancers. Approximativement, $60 \%$ des cancers des VADS sont traités par une chirurgie. L'aggravation du statut nutritionnel a été souvent notée en préopératoire entre la date de l'endoscopie diagnostique et de la chirurgie. Ce statut continue à se dégrader en post opératoire. Selon l'acte chirurgical réalisé, la malnutrition peut s'aggraver par un effet mécanique telle que la genèse d'un néopharynx ou résection d'une partie du tube digestif supérieur (10). A cet effet, s'ajoute le changement du métabolisme post opératoire. En effet, après la chirurgie, il existe des dépenses énergétiques supérieures aux dépenses du métabolisme de base, d'autant plus que dans la chirurgie des cancers des VADS, il existe une modification de la continuité ou une mise au repos d'une partie du tube digestif empêchant ainsi une texture alimentaire normale (11).

La chirurgie peut induire également un effet négatif sur les sens (goût et odorat), la salivation, l'appétit et la psychologie altérant ainsi le désir de s'alimenter (11). En post opératoire, des fausses routes peuvent s'installer obligeant parfois l'arrêt de l'alimentation orale à une période plus longue (3)

De ce fait, une stratégie d'alimentation pré et post opératoire doit être planifiée le plus tôt possible afin de garantir de meilleur résultat. 


\section{- La chimiothérapie}

La chimiothérapie est un traitement systémique qui affecte la totalité du corps. Elle a un effet direct d'aggravation de l'anorexie, changement du goût, déshydratation précoce, nausées et vomissements (12). La chimiothérapie peut ainsi induire un trouble hydro électrolytique et acido basique. La conséquence serait une aggravation du profil nutritionnel. A ces troubles, viennent s'ajouter les complications de la chimiothérapie à type de stomatite et mucite.

En contre partie, la dénutrition peut induire une augmentation de la toxicité des produits de chimiothérapie. En effet, Freyer et al ont trouvé que la dénutrition est le principal facteur de risque de neutropénie fébrile chez le sujet âgé, toxicité particulièrement grave de la chimiothérapie (6).

\section{- La radiothérapie}

L'effet de la radiothérapie sur le tissu sain peut induire des changements dans les fonctions physiologiques du corps ce qui endommage le statut nutritionnel. En effet, la radiothérapie peut entraver plusieurs fonctions notamment l'ingestion, la digestion et l'absorption des aliments. Pour les cancers des VADS, les dégâts sont majeurs puisque la zone à irradier concerne en général la cavité buccale, les muscles masticateurs ou les glandes salivaires. Ces effets dépendent de la structure irradiée, la dose totale, le mode de fractionnement, la durée et le volume irradié. Les complications les plus redoutées et pouvant aggraver le statut nutritionnel sont la xérostomie, l'hyposialie, la stomatite, les contractures musculaires, l'hypogueusie et l'hyposmie. Ces effets commencent généralement à la deuxième ou troisième semaine du début du traitement et diminuent 2 à 3 semaines après la fin de la radiothérapie. Certains effets secondaires peuvent passer à la chronicité tels que les troubles du goût ou de l'odorat.

\section{$\Rightarrow$ Effet du profil du malade porteur de cancer des} VADS sur le statut nutritionnel :

Les cancers des VADS ont comme facteurs de risque majeur l'alcoolo-tabagisme. Ces deux éléments ont un effet négatif sur le statut nutritionnel et pondéral bien établi avant même l'apparition du cancer. En effet, le tabac est responsable d'une élévation du métabolisme de base qui pourrait être liée à une plus grande thermogenèse facultative $(3,13)$. Ce facteur a été rapporté dans certaines séries comme étant un facteur déterminant d'une dénutrition difficile à prendre en charge par voie entérale $(14,15)$. L'éthylisme chronique interfère sur l'absorption et la bio disponibilité de certains micronutriments : acide folique, vitamine $B$, vitamine $C$, manganèse et zinc, ce qui entraîne un déficit en nutriments essentiels et un faible apport énergétique. Associés, alcool et tabac sont res- ponsables d'une réduction majeure de l'appétit avec altération de l'état dentaire réduisant ainsi l'apport alimentaire et modifiant la texture de la ration journalière.

$\Rightarrow$ Immunologie

Les cytokines sont absentes ou indétectables dans le sérum humain. Celles retrouvées dans l'oreille moyenne sont donc produites localement $(6,14)$. Le TGF- $\beta$, puissant facteur chimiotactique pour les leucocytes mononuclés, est retrouvé dans les sécrétions d'une otite séromuqueuse (OSM). Le PAF sécrété peut induire l'OSM même en cas de fonctionnement normal de la trompe d'Eustache (14). Les polynucléaires neutrophiles ont une bactéricidie oxygénodépendante. En détruisant les micro-organismes, ils libèrent des radicaux superoxydes qui pourraient léser la muqueuse de l'oreille moyenne. Cependant, cette muqueuse est protégée par la superoxyde dismutase qui est présente en concentration élevée dans l'effusion muqueuse $(6,7,15)$.

\section{EPIDEMIOLOGIE}

Différentes études ont rapporté que 35 à $50 \%$ des patients pris en charge en ORL pour cancer des VADS souffrent d'une dénutrition. La dénutrition, symptôme de la maladie sous-jacente, est à la fois un indice de gravité du cancer mais aussi un facteur pronostique préthérapeutique aggravant (3). L'influence de l'amaigrissement préthérapeutique sur la mortalité et la morbidité post thérapeutique et particulièrement post opératoire est établie depuis plus d'une soixantaine d'année $(3,16,187)$. L'âge et le sexe n'étaient pas discriminants de façon significative sur la dénutrition en préthérapeutique quoique plus fréquente dans les âges extrêmes et particulièrement chez les sujets âgés (2). II semble que les cancers de la cavité buccale ainsi que de l'hypopharynx altèrent plus rapidement et de façon plus grave le statut nutritionnel que les autres cancers des VADS $(1,11)$.

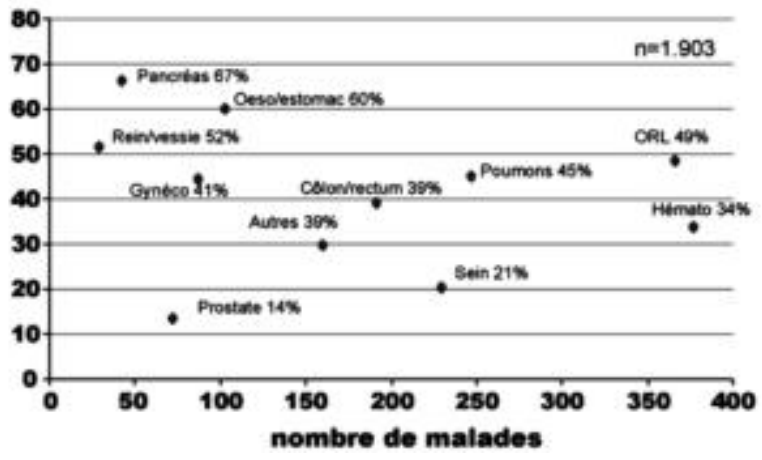

Figure 2 : Prévalence de la dénutrition par localisation de cancer chez 1903 malades (18) 


\section{EVALUATION DE L'ETAT NUTRTIONNEL}

Elle a plusieurs objectifs :

1. Identifier les patients dénutris et ceux qui sont à risque de dénutrition du fait des traitements proposés.

2. Rechercher les principaux facteurs de cette dénutrition et estimer les possibilités de prise en charge.

3. Quantifier le degré de dénutrition et par là le risque pour que le patient développe une complication périthérapeutique.

4. Permettre de suivre et d'évaluer l'efficacité et la tolérance de la prise en charge nutritionnelle.

II serait donc préférable d'avoir des moyens fiables, si possible peu coûteux et non invasifs pour pouvoir les répéter durant la prise en charge.

\section{$\Rightarrow$ L'interrogatoire et l'examen clinique}

L'interrogatoire permet de chercher outre l'évolution pondérale, les modifications récentes des capacités physiques et d'alimentation, les troubles digestifs ou de la déglutition et les douleurs. Une analyse de la ration calorique et protéique par un interrogatoire minutieux est nécessaire pour établir l'équilibre entre les apports alimentaires et les besoins théoriques, afin d'ajuster la prise en charge. Les capacités physiques sont évaluées en cancérologie par l'échelle de l'OMS ou l'échelle de Karnofsky.

L'examen clinique apprécie en particulier la perte de graisse sous cutanée, le degré de fonte musculaire, la présence d'ascite ou d'œdèmes, les signes éventuels de déshydratation

\section{$\Rightarrow$ Mesure du poids, de la perte de poids et de la taille}

Le poids actuel comparé à un poids antérieur à la maladie plutôt qu'à un poids idéal permet d'évaluer l'effet de la maladie sur l'état nutritionnel du malade. Le recueil de la taille est nécessaire afin de calculer I'IMC (indice de masse corporelle). L'IMC est le rapport du poids en $\mathrm{Kg}$ sur le carré de la taille en mètre. L'interprétation du poids n'a de valeur que couplée à un examen clinique et la recherche en particulier d'œdèmes, d'ascite ou de signes de déshydratation. La présence d'œdèmes cliniques signe une expansion du secteur extravasculaire d'au moins $10 \%$, soit généralement plus de $3 \mathrm{~kg}$ et souvent de 5 à $10 \mathrm{~kg}$. C'est surtout la perte de poids et la vitesse de cette dégradation pondérale qui permettent de quantifier le risque. Le pourcentage de perte de poids est le rapport en $\%$ de la perte de poids sur le poids habituel. Une perte de poids de plus de $10 \%$ au cours des 6 derniers mois est un facteur pronostique reconnu au cours de la maladie $(16,19)$. Blackburn et al. (20) définissent une perte de poids sévère (associée à une augmentation significative de la morbidité et/ou de la mortalité) comme $>2 \%$ de perte au cours de la dernière semaine, $>5 \%$ pour le dernier mois, $>7,5 \%$ pour les trois derniers mois, et $>10 \%$ au cours du dernier semestre. Les experts français jugent que $10 \%$ de perte de poids au cours des six derniers mois est un indicateur suffisant de dénutrition préopératoire $(3,21)$.

\section{$\Rightarrow$ Les autres paramètres anthropométriques}

II s'agit de:

- une mesure des plis cutanés, reflet de l'adiposité

- la mesure de la circonférence musculaire brachiale (circonférence à mi-bras en $\mathrm{cm}-[0,314 \mathrm{X}$ (pli tricipital + pli bicipital en $\mathrm{mm} / 2]$ )

Ces deux paramètres sont moins applicables en pratique mais peuvent différencier la perte adipeuse de la fonte musculaire.

\section{$\Rightarrow$ Paramètres biologiques}

L'hypoalbuminémie est associée à un risque de complication et mortalité per et post thérapeutique. Les valeurs seuils d'hypoalbuminémie sévère, de valeur pronostique, varient selon les auteurs de 30 à $35 \mathrm{~g} / \mathrm{L}$. Les autres protéines plasmatiques de demi-vie plus courte, marqueurs de dénutrition (préalbumine, transferrine, protéine vectrice du rétinol), ne peuvent suffire et quantifier le degré du risque de retentissement de la dénutrition sur le pronostic $(22,23)$.

\section{$\Rightarrow$ Scores multiparamétriques}

L'indice le plus simple et de pratique courante est celui de Buzby (NRI= Nutrition Risk Index)

$\mathrm{NRI}=1,519 \times$ (albumine en $\mathrm{g} / \mathrm{L}$ ) $+0,417 \times$ (poids actuel/poids habituel) $\times 100$

\section{$\Rightarrow$ Evaluation nutritionnelle}

Elle comporte deux volets :

- Appréciation des dépenses énergétiques de repos : elle peut être évaluée par calorimètrie indirecte ou calculée par des formules

- Analyse de la ration calorique et protéique qui permet d'établir l'équilibre entre les apports alimentaires et les besoins théoriques.

En pratique, une hiérarchie d'évaluation est bien établie (fig.3) 


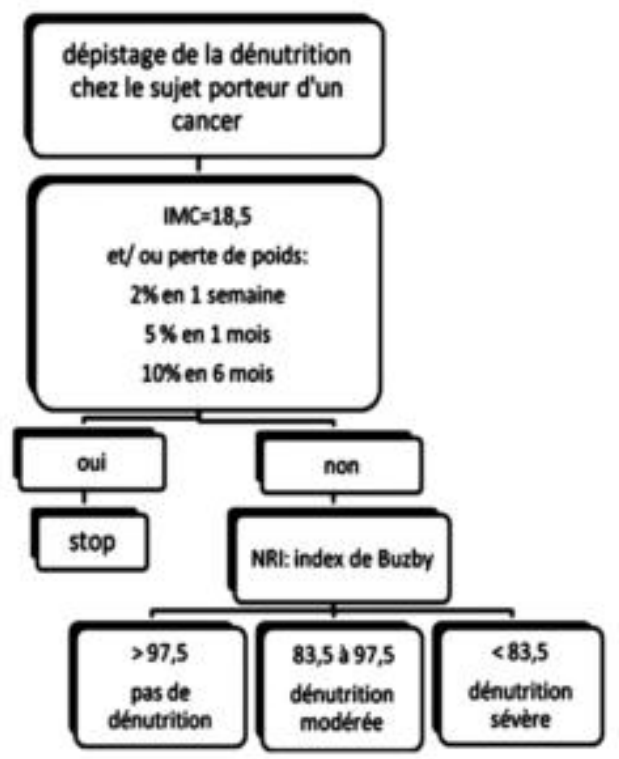

Figure 3 : Arbre décisionnel du dépistage de la dénutrition chez un malade porteur d'un cancer (24)

\section{PRISE EN CHARGE NUTRITIONNELLE EN PRÉ THÉRAPEUTIQUE}

La dénutrition préthérapeutique majore le risque de complications post opératoires et peut à elle seule contre indiquer l'indication d'un traitement curatif à base de chimiradiothérapie (25).

Faisant suite à plusieurs études portant sur un nombre important de malades, lors de la conférence de consensus sur la chirurgie programmée de l'adulte, il a été recommandé que les patients présentant une dénutrition sévère (IMC < 18,5; NRI < 83,5 et / albuminémie < 30g/L) et devant subir un traitement lourd devraient bénéficier d'une nutrition préopératoire si possible (sans retarder le traitement) et post opératoire systématique. Chez de tels patients ayant une morbidité révélée en préthérapeutique, le rapport bénéfices/risque est en effet clairement favorable $(1,26,27,28)$.

Cette position a été reprise et confirmée par les recommandations de la société américaine de nutrition entérale et parentérale (1).

La voie orale est en règle suffisante. Elle peut être directe ou via une sonde nasogastrique. Les objectifs caloriques (30 à $35 \mathrm{Kcal} / \mathrm{kg} / \mathrm{j}$ ) devront être atteints progressivement par palier de 15 jours. Les 3 premiers jours, un apport de 1000 cal est suffisant et justifié. Si l'objectif nutritionnel ne peut pas être atteint par voie entérale, en raison de complications ou d'intolérance, le recours à une nutrition mixte, entérale et parentérale peut être justifié. Les apports caloriques recommandés doivent être assurés par l'association de glucides (40 à $60 \%$ des apports énergétiques totaux) de lipides (20 à $40 \%$ ) et de protéines (15 à 25\%). Chez les patients particulièrement dénutris, la rénutrition devra être prudente, enrichie en phosphore et en vitamines avec un apport initial équivalent à celui ingéré au préalable par le patient afin d'éviter le « refeeding syndrom » souvent mortel $(1,21,27,29)$.

\section{PRISE EN CHARGE NUTRITIONNELLE EN POST OPERATOIRE}

L'alimentation entérale est reprise dès les 24 heures postopératoires dans le cas d'intégrité des voies digestives. Dans le cas contraire, elle sera assurée par sonde nasogastrique. Cette alimentation doit être progressive commençant par $500 \mathrm{cc}$ de sérum glucosé à $5 \%$ le 1 er jour pour tester la tolérance mécanique. L'apport caloricoazoté nécessaire est calculé sur la base des besoins au repos donnée par la loi de Harris et Benedith majorée de $20 \%$ (sujet agressé) avec un apport azoté de 0,2 $\mathrm{g} / \mathrm{kg} / \mathrm{jour}$. Le taux calorique trouvé lors de l'enquête alimentaire préopératoire sera administré au 2ème jour post opératoire suivi d'une augmentation de 100 à 200 calories/jour et de 5 à 10 grammes de protéines/j jusqu'à atteindre l'apport nécessaire calculé par la formule de Harris $(21,27,30)$.

\begin{tabular}{|c|c|c|c|}
\hline & Quand? & Comment? & Avec quoi? \\
\hline Pre-cpetratoire & $\begin{array}{l}\text { Au moins } 7 \\
\text { jours avant } \\
\text { chinurgie }\end{array}$ & $\begin{array}{c}\text { Orale ou } \\
\text { entérale+t+ (SNG ou } \\
\text { gastrostomie) } \\
\text { A domicile si possible } \\
\text { Parenterale si } \\
\text { necessaire }\end{array}$ & $\begin{array}{l}\text { Immunonutriton } \\
\text { riche en arginine et } \\
\text { acides gras } n-3\end{array}$ \\
\hline Post operatoire & $\begin{array}{l}\text { Precoce chez le } \\
\text { patient dónutri } \\
\text { ( } 24 h \text { ) tant que } \\
\text { les ingesta } \\
\text { restent < } 1000 \\
\text { KcalJ }\end{array}$ & $\begin{array}{l}\text { Entitrale en premiere } \\
\text { intention par sonde } \\
\text { naso-gastrique, } \\
\text { gastrostomie ou } \\
\text { jejunostomie }\end{array}$ & $\begin{array}{l}\text { Timmunonutrition du } \\
\text { mème type si } \\
\text { dénutrition pré- } \\
\text { operatoire }\end{array}$ \\
\hline
\end{tabular}

Tableau II : Recommandations pour la mise en œuvre d'une nutrition péri-opératoire chez le malade cancéreux (31)

\section{PRISE EN CHARGE NUTRITIONNELLE ET RADIO-CHIMIOTHERAPIE}

La mise en œuvre d'une assistance nutritionnelle au cours de la radio-chimiothérapie parait d'autant plus logique que ces traitements sont bien connus pour leurs effets secondaires, en particulier digestifs, et donc pour le risque inhérent de majoration de la dénutrition. En effet, il est bien établi qu'une perte de poids moyenne de 4 à $5 \mathrm{Kg}$ durant la radiothérapie des cancers des voies aéro-digestives supérieures est habituelle en l'absence de suivi nutritionnel particulier (32). En outre, la prévention ou la correction de la dénutrition devrait être de nature à optimiser les résultats du schéma thérapeutique en limitant la nécessité de réduction de doses ou de retard de cures. 
Tant que la voie orale peut assurer l'apport nutritionnel adéquat à la perte énergétique, elle sera privilégiée avec une surveillance pondérale bihebdomadaire. À la moindre dégradation de l'état nutritionnel, il est recommandé de recourir à la voie entérale par sonde nasogastrique, gastrostomie ou jéjunostomie. Le bénéfice avec la moindre morbidité de la voie entérale semble supérieur à la voie parentérale. En effet, cette dernière est associée à un taux de surinfection du cathéter central non négligeable avec une efficacité comparable. II a été également évoqué que la voie parentérale avait un effet stimulant des cellules tumorales. Cependant, la voie parentérale garde une place indiscutable pour les patients sévèrement dénutris si on anticipe que le patient serait incapable de se nourrir pour une durée supérieure à 7 jours. Elle peut également être indiquée après gastrostomie en attendant la fonctionnalité de cette sonde $(4,9)$.

\section{CONCLUSION}

Etat morbide fréquent, la dénutrition mérite d'être cherchée systématiquement et diagnostiquée précocement chez tout porteur d'un cancer des voies aéro-digestives supérieures afin d'améliorer l'observance et lé tolérance du traitement curatif.

Une simple quantification staturo-pondérale permettra d'individualiser une population particulièrement dénutrie, à risques graves, devant bénéficier d'une prise en charge pré-thérapeutique. Celle-ci s'impose alors quelque soit le programme thérapeutique envisagé, (chirurgie, radiothérapie) en pré, per, voire post-thérapeutique. Un suivi sérieux par une diététicienne permettra d'orienter les autres patients vers une prise en charge médicale dès que nécessaire.

\section{REFERENCES}

1. Déchelotte, P. . Prise en charge de la dénutrition chez le patient cancéreux. Nutr Clin Métabol, 2000;14: 241-9.

2. Van den Berga M.G.A., Rasmussen-Conrada E., Gwasaraa G, Krabbeb P., Naberc J, Merkxe M. A prospective study on weight loss an energy intake in patients with head and nekh cancer, during diagnosis, treatment and revalidation. Clin Nutr. $2006 ; 25: 765-772$

3. Wiel E., Costecalde M, Séguy D., Merrot O., Erb C., Chevalier D. et al. Evolution périopératoire du statut nutritionnel en chirurgie carcinologique des voies aérodigestives supérieures. Etude prospective et descriptive. Ann $\mathrm{Fr}$ Anesth Réanim. $2005 ; 24: 600-606$.

4. F. Bozzetti , J. Arends , K. Lundholm , A. Micklewright, G. Zurcher , M. Muscaritoli. ESPEN guidelines on parenteral nutrition: non surgical oncology. Clin Nutr . $2009 ; 28: 445-454$

5. Tesdale MJ. Pathogenesis of canecr cachexia,. J Support Oncol. 2003 ; 1 : $159-68$.

6. Freyer G, Geay J., Touzet S. Comprehensive geriatric assessement predicts tolerance to chemotherapy and survival in elderly patients with advanced ovarian carcinoma. Ann Oncol. $2005 ; 16: 1795-800$.

7. Nitenberg G. Physiopathologie de la dénutrition chez le cancéreux. Nutr. Clin. Métabol ;1997; 11 (4, suppl 1) : 364-372.

8. Ramos E J, Suziki S., Marks D. Cancer anorexia-cachexia syndrome: cytokines and neuropeptides. Curr Opin Clin Nutr Metabol Care. $2004 ; 7$ (4) : 427-34. 9. Arends J., Bodoky G., Bozzoti F., Feraon K., Muscaritoli M. Selga G. et al. ESPEN guidelines on enteral nutrition: non surgical oncology. Clin Nutr. 2006 ; $25: 245-259$.

10. Guire M. Nutritional care of surgical oncology patients. Semin Oncol Nurs. $2000 ; 16$ (2) : 128-34.

11. Vanbokhorst-de vander schueren M, Langendoen S., Vondeling H., Kuik D, Quak J, Janleeuwen A.Perioperative enteral nutrition and quality of life ofseverely malnourished head and neck cancer patients: a randomized clinical trial. Clin Nutr. $2000 ; 19$ (6) : 437-444.

12. Fishman M, Mrozek Orlovski . Cancer chimiotherapy Guidelines and recommandations for practice. Pittsburgh: Oncology Nursing Press 1999.

13. Wood MR., Lander V., Mosby L., Hiatt R. Nutrition and the head and neck cancer patient. Oral Surg Oral Med Oral Pathol . $1989 ; 68: 391-5$

14. Thuong M., Leteurtre S., Recommandations aux experts de la société de réanimation de langue frnçaise : nutrtion entérale en réanimation. Réanimation. $2003 ; 12: 350-354$.

15. Mangar S., Selven N., Mais K., Sykes A. Evaluating predictive factors for dtermining enteral nutrition in patients receiving radical radiotherapy for head and neck cancer: a retrospective review. Radiotherapy and oncology . $2006 ; 78$ : 152-158.

16. Costanzo J. Influence de l'état nutritionnel préopératoire sur la morbidité postopératoire. Nutr Clin Métabol. 1995 ; 9 (Suppl 1) : 39-44.
17. Studely HO. Percentage of weight loss: a basic indcator of surgical risk in patients with chronic peptic ulcer. JAMA. 1936 ; $106: 458-60$

18. Hébuterne $X$, Lemarié $E$., Michallet $M$. Prévalence de la dénutrition au cours du cancer : une enquête. Nutr Clin Metab. 2006 ; 20 : S 86.

19. Dewys WD, Begg C., Lavin P. Prognostic effect of weight loss prior to chemetherapy in cancer patients. Am J Med. $1980 ; 69: 491-7$.

20. Blackburn GL, Bistrian B., Maini B. Nutritional and metabolic assessment of the hospitalized patient. JPEN. 1977: 11-12.

21. Bachmann, P. Prise en charge nutritionnelle périopératoire. nutrition clinique et métabolisme. $2008 ; 22: 33-38$

22. Bachmann, P. Nutrition et soins palliatifs en cancérologie. Nutrtion clinique et métabolisme. $2006 ; 20: 137-141$.

23. Guérin $O$. Prise en charge nutrtionnelle en oncogériatrie. Nutrtion clinique et métabolisme. $2009 ; 23: 16-18$

24. Bachmann P, Marti C., Vincent B. Standards, Options and recommandations for nutritional support in adult patients with advanced or terminal cancer . Bull Cancer .2001; $88: 985-1006$.

25. Senesse $P$, Assenat E, Schneider S, Chargari C, Magné N, Azria D et al. Nutritional support during oncologic treatment of patients with gastrointestinal cancer: Who could benefit?.Cancer Treatment Reviews. 2008 ; 34(6) : 568-575. 26. Arends J., Bodoky G., Bozzetti F., Fearon K., Muscaritoli M., Selga G. et al. Epsen guidelines on enteral nutrition: non surgical oncology. Clin Nutr. 2006 ; 25 : 245-59.

27. Petit J., Zazzo J. . Evaluation de la nutrtition entérale: aspects clinique et paraclinique, aspect médico-économique, démarche - qualité. Réanimation.2006; 12 : 387-392.

28. Hébuterne $X$. La lettre du président : nutrition du malade cancéreux : il est temps d'agir. Nutrition clinique et métabolisme. $2008 ; 22: 1-2$

29. Jemaa Y.,Lecleire S,Petit A, Déchelotte P. Prise en charge nutritionnelle périopératoire en chirurgie de l'adulte. Nutrition Clinique et Métabolisme. 2004;18(3) : 137-146.

30. Rethinking D, Ottery. F. Nutritional support of the cancer patient: The new field of nutritional oncology. semin oncol. $1994 ; 21: 770-778$.

31. Nitenberg, G. Nourrir le patient cancéreux en 2006: aujourd'hui mieux que jadis? Nutrition clinique et métabolisme. $2006 ; 20: 549-554$.

32. Garabige V., GiraudP, De Rycke $Y$, Girod A, Jouffroy $T$, Jaullery C. Intérêt de la prise en charge nutritionnelle des patients traités par irradiation des voies aéro-digestives supérieures: la consultation diététique est -elle utile? Cancer Radiotherapy. 2007 ; 11 :111-116. 